\title{
A NOTE ON CENTRAL GROUP EXTENSIONS
}

\author{
G. J. HAUPTFLEISCH
}

(Received 3rd May 1971)

Communicated by G. E. Wall

If $A, B, H, K$ are abelian groups and $\phi: A \rightarrow H$ and $\psi: B \rightarrow K$ are epimorphisms, then a given central group extension $G$ of $H$ by $K$ is not necessarily a homomorphic image of a group extension of $A$ by $B$. Take for instance $A=Z(2)$, $B=Z \oplus Z, H=Z(2), K=V_{4}$ (Klein's fourgroup). Then the dihedral group $D_{8}$ is a central extension of $H$ by $K$ but it is not a homomorphic image of $Z \oplus Z \oplus Z(2)$, the only group extension of $A$ by the free group $B$.

In [1] is is shown that there exists a certain class of loops $L$ which can be considered to be extensions of an abelian group $A$ by a group $B$. It turns out that this loop extension theory contains the Hölder-Schreier theory as a special case. The purpose of the present note is to show that if we have the above situation in this more general extension theory, we can prove the following:

THEOREM. Any central group extension $G$ of $H$ by $K$ is a homomorphic image of a central loop extension $L$ of $A$ by $B$.

Before we prove this theorem, let us recall some definitions:

If $C$ is any subgroup of $A$, then a function $f: B \times B \rightarrow A$ is called a $(B, A, C)$ quasi factor system if it satisfies the following conditions for all $a, b, c \in B$ :

(1) $f(b, 0)=f(0, b)=0$

(2) $f(a+b, c)+f(a, b)-f(a, b+c)-f(b, c) \in C$

(3) $f(-b, b)=f(b,-b)$.

Note that if $C=0$, then $f$ is a factor system.

Given abelian groups $A$ and $B, C$ a subgroup of $A$ and a $(B, A, C)$-quasi factor system $f$, then the cartesian product $L=B \times A$ with the operation

$$
(b, a)+\left(b^{\prime}, a^{\prime}\right)=\left(b+b^{\prime}, a+a^{\prime}+f\left(b, b^{\prime}\right)\right)
$$

is a central loop extension of $A$ by $B$.

If $L$ is an arbitrary central loop extension of $A$ by $B$ with associated quasi factor system $f$ and $G$ is any central group extension of $H$ by $K$ with associated factor system $g$, then it follows directly that the mapping

$$
\theta: L \rightarrow G ;(b, a) \theta=(b \psi, a \phi)
$$


is an epimorphism if and only if

$$
g\left(b \psi, b^{\prime} \psi\right)=\left\{f\left(b, b^{\prime}\right)\right\} \phi \text { for all } b, b^{\prime} \in B .
$$

We now proceed to prove the theorem.

Proof. Consider an arbitrary central group extension $G$ of $H$ by $K$ with associated factor system $g$. Let $\operatorname{Ker} \phi=C$ and let $\Phi$ be the isomorphism $A / C \cong H$. We now construct a loop extension $L$ of $A$ by $B$ such that $G$ is a homomorphic image of $L$.

To this end, select representatives $r(h)$ in the cosets modulo $C$ in $A$ corresponding to $h \in H$ under $\Phi$, and select, in particular, 0 in $C$.

Define $f: B \times B \rightarrow A ; f\left(b, b^{\prime}\right)=r\left\{g\left(b \psi, b^{\prime} \psi\right)\right\}$.

It is clear that $f$ satisfies (1).

To show that $f$ satisfies (2), we consider arbitrary $b, b^{\prime}, b^{\prime \prime} \in B$. Since $g$ is a factor system, we have

$$
g\left(b \psi+b^{\prime} \psi, b^{\prime \prime} \psi\right)+g\left(b \psi, b^{\prime} \psi\right)-g\left(b \psi, b^{\prime} \psi+b^{\prime \prime} \psi\right)-g\left(b^{\prime} \psi, b^{\prime \prime} \psi\right)=0 .
$$

Going over to $A / C$ under $\Phi$, we have the required result. Finally,

$$
f(-b, b)=r\{g(-b \psi, b \psi)\}=r\{g(b \psi,-b \psi)\}=f(b,-b) .
$$

Thus, $f$ is a $(B, A, C)$-quasi factor system and so $L=B \times A$, with the operation (4), is a central loop extension of $A$ by $B$.

By definition, $f$ satisfies (6) and so the mapping $\theta$ defined by (5) is the required epimorphism.

\section{Reference}

[1] G. J. Hauptfleisch, 'Quasi-group extensions of Abelian Groups' (Thesis, Leiden, 1965.)

Tulane University

New Orleans

Louisiana, U.S.A.

and

Rand Afrikaans University

Johannesburg

Republic of South Africa 